\title{
14
}

\section{The impact of air pollution on mortality in Shanghai}

\section{Health and Mortality Transition in Shanghai Project Research Team}

Climate change, air pollution and improving public health are some of the most serious challenges facing humankind at the beginning of the twenty-first century. While considerable effort has been made to understand the impact of changing climatic conditions and air quality on population health, our knowledge of their relationship is still rather limited, and this is especially the case in developing countries, where detailed and reliable data for environmental conditions and population health are often difficult to find.

To further improve our knowledge of declining mortality rates in the past half-century, the major characteristics of current mortality patterns and their future changes in China, researchers from The Australian National University, Fudan University, Shanghai Municipality Centre for Diseases Control and Prevention and the University of Cambridge have been conducting a detailed study of health and mortality transition in Shanghai. This project, which has been funded by a research grant from the Wellcome Trust, aims to achieve the following objectives: to provide detailed information about changes in causes of death and mortality levels in Shanghai in recent decades, to gain a better understanding of changing mortality patterns and their relationship with major socioeconomic and environmental factors, and to further examine the process of China's epidemiological transition and some proposed mortality patterns and their changes.

The project has collected and digitalised detailed population and mortality data collected from seven urban districts in Shanghai from the late 1950s to the beginning of the twenty-first century. In addition, it has gathered detailed 
information on weather for the past four decades and air-quality data for recent years. These data are used in this study to explore the relationship between air pollution, weather and mortality in Shanghai.

Shanghai, like many large cities in developing countries, has been experiencing a rapid transition. Fast economic growth and urban expansion have led to considerable changes in people's living environments, which in turn could greatly influence improvements in population health and socioeconomic development. The impact of changing air quality and climate on population health is an issue that has attracted great attention in recent years. Compared with some other Chinese cities, Shanghai's air quality has been reasonably good; however, in comparison with industrialised countries and the air-quality guidelines recommended by the World Health Organization (WHO), there is still a long way to go to control air pollution in Shanghai (Brajer and Mead 2004; Kan et al. 2007; WHO 2005). For example, out of 3,149 days between 1998 and 2007, when the data were available, the level of particulate matter less than 10 micrometres $(\mu \mathrm{m})$ in aerodynamic diameter $\left(\mathrm{PM}_{10}\right)$ reached the standard recommended by the WHO air-quality guidelines on only 29.1 per cent of days; the levels of nitrogen dioxide and sulphur dioxide reached their standards on 50 per cent and 10 per cent of the days, respectively. The situation was worse in cool seasons when the proportion of days when these pollutants were lower than or reached the level recommended by the WHO guidelines fell to 23.9 per cent (for $\mathrm{PM}_{10}$ ), 39.2 per cent (for nitrogen dioxide) and 6.8 per cent (for sulphur dioxide). The proportion of days when the levels of these three pollutants all reached the WHO guidelines was even lower: about 5 per cent.

An increasing number of studies exploring the link between air pollution and population health have been conducted in recent years, and they have shown that air pollution has considerable effects on morbidity and mortality in most of the populations being investigated (Kenney and Ozkaynak 1991; Xu et al. 1994, 2000; Ostro et al. 1996; Touloumi et al. 1997; Lee et al. 1999; Wong et al. 2001; Kan et al. 2003, 2007). Despite this, there is still considerable uncertainty about the nature and the strength of such effects, which is partly attributable to the following facts. First, the number of studies, particularly those conducted in developing countries, is still limited. Second, most available single-city studies use different statistical models. Because real time-series data such as air quality and mortality often have greater complexity than many commonly used models allow, conclusions drawn from these studies tend to be sensitive to model specification (Erbas and Hyndman 2005).

To improve our knowledge about the relationship between air quality and population health, especially in the context of rapid urban development in 
developing countries, this study systematically analyses the impact of air pollution on mortality in Shanghai. On the basis of this analysis, it also compares the impact of air pollution on daily mortality between Shanghai and Hong Kong-two major cities in Asia.

\section{Data and methods}

Mortality data collected by Shanghai Municipality Centre for Diseases Control and Prevention are used in this study. With financial support provided by the Wellcome Trust, the Health and Mortality Transition in Shanghai Project has digitalised nearly 300,000 death certificates issued between 1956 and 2001 in seven urban districts: Nanshi, Luwan, Xuhui, Changning, Zhabei, Hongkou and Yangpu. These death certificates record the following information for each deceased: name, sex, date of birth, date of death, race, personal ID number, home address, educational level, occupation, marital status, age at death, the principle cause of death and place of death (although there are variations in the amount of information included over time). They also record the level of the hospital (provincial, county or township) that certified the death, major methods of diagnosis (autopsy, biopsy, laboratory test, clinical diagnosis or inference on the basis of available evidence) and other useful information. In this study, deaths are classified into groups according to the International Classification of Diseases Revision Nine (ICD-9). Since information on the date of death is available, daily mortality patterns can be examined.

The meteorological data for Shanghai between 1956 and 2001 are extracted from a data set made available by China's Meteorological Bureau. They provide information on daily average air temperature, relative humidity and atmospheric pressure for most years. The collection of air-quality data started late in Shanghai. Detailed information of levels of major pollutants such as $\mathrm{PM}_{10}$, sulphur dioxide and nitrogen dioxide, which can be accessed directly from the web site of the Shanghai Environmental Protection Bureau, became available only from the late 1990s. For this reason, our analysis of the relationship between air quality and mortality has to be restricted to the years 2000 and 2001 when detailed data for mortality, weather and air quality were all available.

Since one of the objectives of this chapter is to compare the impact of air pollution on daily mortality between Shanghai and Hong Kong, we have chosen to use the method used in Wong et al. (2001). In the analysis, Poisson regression was used with daily mortality counts as the dependent variable. To obtain the core model for the mortality outcomes in which we are interested, smoothed (through the use of the Loess smoothing function) terms for trends on days, seasonality, temperature, humidity and dummy variables for days 
of the weeks were fitted as the independent variables. Like Wong et al., we considered lag effects of temperature and humidity, but other factors such as the effects of holidays and influenza epidemics (measured by a large number of hospital admissions for influenza) were not included in our estimation. A detailed discussion of the method can be found in Wong et al. (2001).

The analysis was conducted in the following steps. After obtaining expected mortality counts $(\xi)$ from the core model, Poisson regression was fitted on pollutant concentrations in the form of

$$
\log \left[E\left(Y_{t}\right)\right]-\log \left(\xi_{t}\right)=\alpha+\beta x_{\tau}
$$

to obtain the log relative risk ( $\beta$ ) estimated with offset on log $(\xi)$. In examining the impact of each pollutant on mortality, we estimated the effect of pollutant concentration for the current and previous days, and identified the best lagged day by 'Akaike's information criterion' (AIC). (We also examined the effect of the average level of pollutant concentration on the current and previous days; these results correlate highly with those estimated from the pollutant level for a single day and are not reported here.) The effect of each pollutant was then adjusted for auto-correlation using general least square regression and for the co-pollutant, which was determined using linear regression.

We then further estimated the effect of pollutant concentration on mortality in warm and cold seasons and the effect of the interaction between the season and the pollutant being examined. To make the division of seasons consistent with those used in Wong et al. (2001), we included the six months from October to March in the cold season, and those from April to September in the warm season. Because Shanghai's climatic conditions differ from those in Hong Kong, it might be more appropriate to divide the seasons in different ways; this has been examined in our analysis, but the results are not reported here. When estimating the effect of a single pollutant, we used the same model as that described above but with two additional independent variables: a dummy variable for the season ( 1 for the warm season and 0 for the cold season) and a variable representing the interaction between the season and the pollutant. We then add two more variables - the concentration of the co-pollutant identified earlier and the interaction of the co-pollutant and the season-into the model to estimate the effect of the major pollutant adjusting for the effect of the co-pollutant. In the analysis, we have further examined the exposure-response relationship between the concentration level of the pollutant and mortality outcomes in warm and cold seasons, although detailed results will not be discussed in this chapter. All analyses were conducted using the statistical software $R$. 


\section{Results}

Table 14.1 presents summarised statistics of mortality counts, weather and major pollutants for the study period, by the two seasons specified earlier. While this seasonal division might not be the best way to group months, it has been adopted to make the analysis consistent with that used in the Hong Kong study. Alternative ways of dividing seasons have been considered in the data analysis, but their influence on the results is relatively small.

\section{Table 14.1 Summary statistics of mortality outcome, air pollution levels and meteorological measures by season}

\begin{tabular}{|c|c|c|c|c|c|c|c|c|}
\hline & No. (days) & Mean & $\begin{array}{l}\text { Standard } \\
\text { deviation }\end{array}$ & Minimum & $P_{10}$ & Median & $\mathrm{P}_{90}$ & Maximum \\
\hline \multicolumn{9}{|c|}{ Mortality counts } \\
\hline \multicolumn{9}{|c|}{ Non-accidental } \\
\hline Cool & 365 & 110.2 & 18.5 & 62 & 86 & 109 & 134 & 164 \\
\hline Warm & 366 & 85.3 & 11.2 & 58 & 72 & 84 & 101 & 119 \\
\hline \multicolumn{9}{|c|}{ Cardiovascular disease } \\
\hline Cool & 365 & 40.1 & 9.3 & 16 & 28 & 39 & 53 & 65 \\
\hline Warm & 366 & 28.6 & 5.9 & 14 & 21 & 28.5 & 36 & 50 \\
\hline \multicolumn{9}{|c|}{ Respiratory disease } \\
\hline Cool & 365 & 13.9 & 5.5 & 3 & 7 & 13 & 21 & 32 \\
\hline Warm & 366 & 8.5 & 3.4 & 2 & 4 & 8 & 13 & 22 \\
\hline \multicolumn{9}{|c|}{ Air pollution concentrations $\left(\mu \mathrm{g} / \mathrm{m}^{3}\right)$} \\
\hline \multicolumn{9}{|c|}{ Nitrogen dioxide } \\
\hline Cool & 365 & 61.9 & 36.1 & 20 & 32 & 48 & 100 & 222 \\
\hline Warm & 366 & 43.6 & 29.5 & 13 & 20 & 34 & 82 & 190 \\
\hline \multicolumn{9}{|c|}{ Sulphur dioxide } \\
\hline Cool & 365 & 44.8 & 16.2 & 12 & 24 & 45 & 67 & 98 \\
\hline Warm & 366 & 37.6 & 13.2 & 10 & 20 & 38 & 55 & 74 \\
\hline \multicolumn{9}{|l|}{$\mathrm{PM}_{10}$} \\
\hline Cool & 365 & 73.9 & 45.7 & 9 & 33 & 62 & 131 & 358 \\
\hline Warm & 366 & 65.1 & 30.5 & 16 & 38 & 59.5 & 95 & 338 \\
\hline \multicolumn{9}{|c|}{ Meteorological measurements: } \\
\hline \multicolumn{9}{|c|}{ Temperature $(\stackrel{\circ}{ } \mathrm{C})$} \\
\hline Cool & 365 & 10.6 & 5.9 & -2.1 & 3.6 & 9.8 & 20.1 & 24.4 \\
\hline Warm & 366 & 23.8 & 5.1 & 9.8 & 16.3 & 24.2 & 30 & 33.2 \\
\hline \multicolumn{9}{|c|}{ Humidity (\%) } \\
\hline Cool & 365 & 74.5 & 12.9 & 36 & 57 & 76 & 92 & 99 \\
\hline Warm & 366 & 77.5 & 10.9 & 46 & 62 & 79 & 90 & 98 \\
\hline
\end{tabular}

Sources: Authors' calculations. 
Shanghai is located on China's east coast and has a subtropical monsoon climate. As shown in Table 14.1, Shanghai's average air temperature was 23.8 degrees Celsius $\left({ }^{\circ} \mathrm{C}\right)$ in the warm months and $10.6{ }^{\circ} \mathrm{C}$ in the cold months in 2000 and 2001 -about $3{ }^{\circ} \mathrm{C}$ and $8{ }^{\circ} \mathrm{C}$ lower than the average temperatures recorded in Hong Kong in the two respective seasons over the period from 1995 to 1997. Shanghai has a greater seasonal difference in air temperature than Hong Kong. In Shanghai, the average relative humidity for the cold months was close to that for the warm months. These levels and their seasonal difference are similar to those observed in Hong Kong.

Statistics for pollutant concentrations are presented in the middle panel of Table 14.1. In comparison with warm seasons, in cold seasons, higher levels of concentration of all three listed pollutants were found. The average levels of air pollution in the cold and warm seasons are 61.9 and 43.6 micrograms $(\mu \mathrm{g})$ per cubic metre for nitrogen dioxide, 44.8 and $37.6 \mu \mathrm{g} / \mathrm{cubic}$ metre for sulphur dioxide and 73.9 and $65.1 \mu \mathrm{g} /$ cubic metre for $\mathrm{PM}_{10}$, respectively. The comparison between these results and those recorded in Hong Kong between 1995 and 1997 suggests that, in Shanghai, the level of nitrogen dioxide was slightly lower than in Hong Kong, but the level of sulphur dioxide was much higher, and their seasonal difference was greater than that observed in Hong Kong. In Shanghai, the level of $\mathrm{PM}_{10}$ was also markedly higher than in Hong Kong, especially in the warm months. Another notable difference found between the two cities is that Shanghai's maximum levels of nitrogen dioxide and $\mathrm{PM}_{10}$ are much higher than those recorded in Hong Kong.

In Shanghai, a larger number of non-accidental deaths took place in cold seasons than in warm seasons; this is particularly true for deaths caused by cardiovascular and respiratory diseases. The ratios of cold-month to warmmonth deaths for the three mortality groups specified in Table 14.1 were 1.29 (non-accidental deaths), 1.40 (cardiovascular disease) and 1.64 (respiratory disease). These ratios were higher than those recorded in Hong Kong between 1995 and 1997, which were 1.17 (non-accidental deaths), 1.34 (cardiovascular disease) and 1.15 (respiratory disease).

Table 14.2 presents statistical results showing the effect of listed pollutants on the number of all non-accidental deaths and the number of deaths due to cardiovascular and respiratory diseases. These pollutants and the three types of deaths are listed in the first column of the table. The second column of the table presents the lag day, which is the day on which the level of the given pollutant has the strongest association with the number of deaths recorded on the day of observation. We compared the lag effects for up to three days, and the best single lagged day was identified by the AIC. 


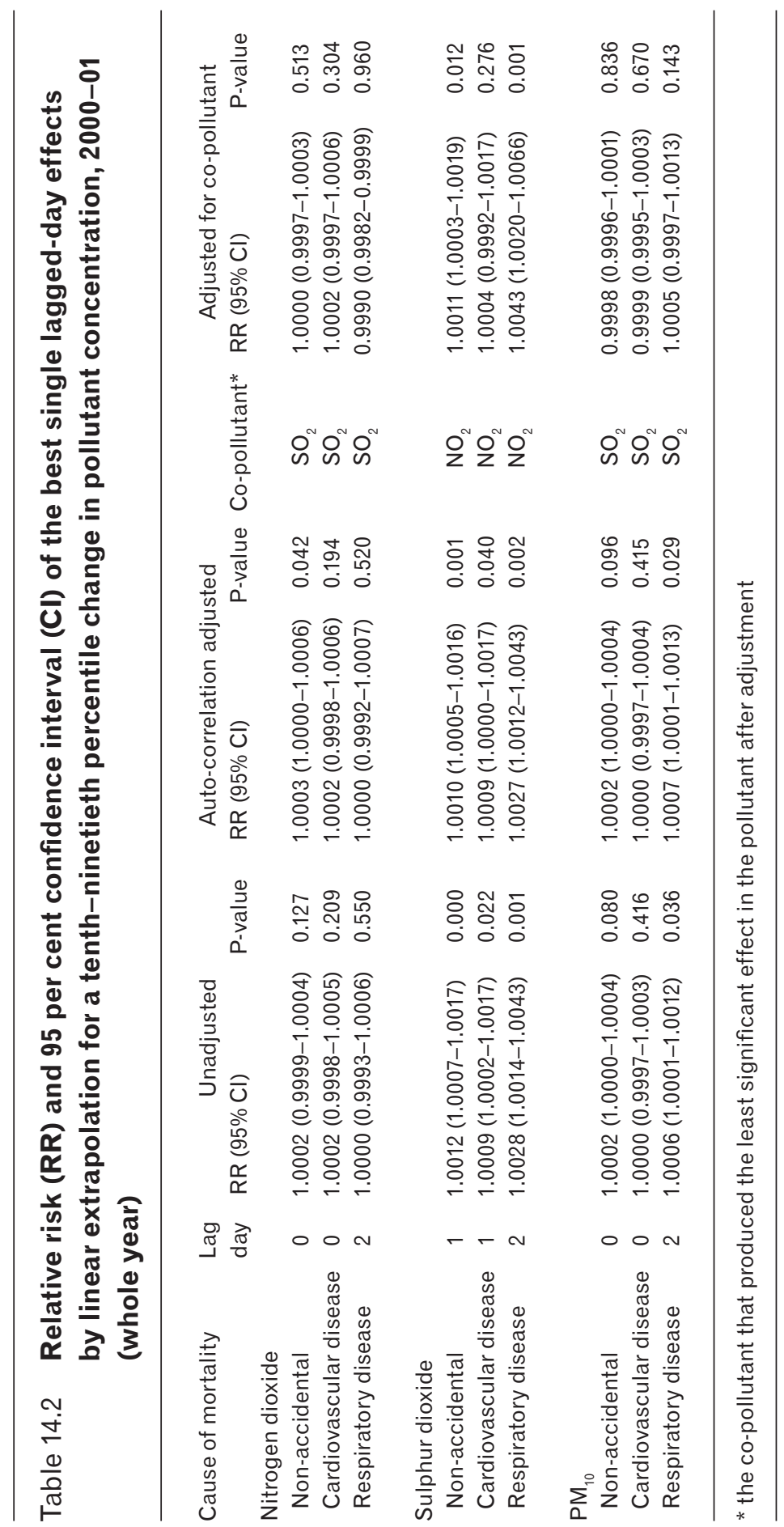


The effect of the given pollutant on mortality outcomes, in the form of relative risks (RRs) and their 95 per cent confidence interval $(\mathrm{Cl})$, is shown in column three and its significant level is shown in column four. These results show that the concentration of sulphur dioxide on the best single lagged day is significantly associated with all three mortality outcomes on the day of observation, and the levels of $\mathrm{PM}_{10}$ are significantly related to the deaths caused by respiratory diseases. These results are not, however, adjusted for auto-correlation and co-pollutants. After adjusting for auto-correlation (using the general least square method made available by the statistical software $R$ ), some changes were observed in the results-shown in columns five and six of the table. These changes are generally small, indicating that the influence of auto-correlation on the results is moderate or weak. We then further adjusted the model to take into account the impact of co-pollutants (listed in column seven), which were identified through the use of linear regression. These results are presented in columns eight and nine. After the model has been adjusted for auto-correlation and co-pollutants, the effects of nitrogen dioxide and $\mathrm{PM}_{10}$ on mortality outcomes are statistically insignificant. The concentration of sulphur dioxide is, however, still associated significantly with the number of all nonaccidental deaths and those caused by respiratory diseases. These results are broadly similar to those in Wong et al. (2001).

Table 14.3 presents statistical results for the effects of specified pollutants on mortality outcomes in the warm and cold seasons, which have been estimated using a model similar to that described above. To estimate the impact of a single pollutant without taking into account the effect of the co-pollutant, two additional variables - a dummy season variable and the interaction between the given pollutant and the season-were added into the model. When the impact of the co-pollutant was adjusted, two further variables-the copollutant and the interaction of the co-pollutant and the season-were also included in the model. These results show that, in the warm season, the effect of all listed pollutants on mortality outcomes is not statistically significant, regardless of whether the effect of the co-pollutant is adjusted. In the cold season, however, the concentration of nitrogen dioxide and sulphur dioxide is related significantly to all mortality outcomes if the effect of the co-pollutant is not adjusted. After the impact of the co-pollutant was taken into account, most of these significant associations remained except that between the concentration of nitrogen dioxide (after being adjusted for the co-pollutant sulphur dioxide) and the number of deaths caused by respiratory diseases. There is no significant association between the concentration of $\mathrm{PM}_{10}$ and specified mortality outcomes, regardless of whether the model was adjusted 


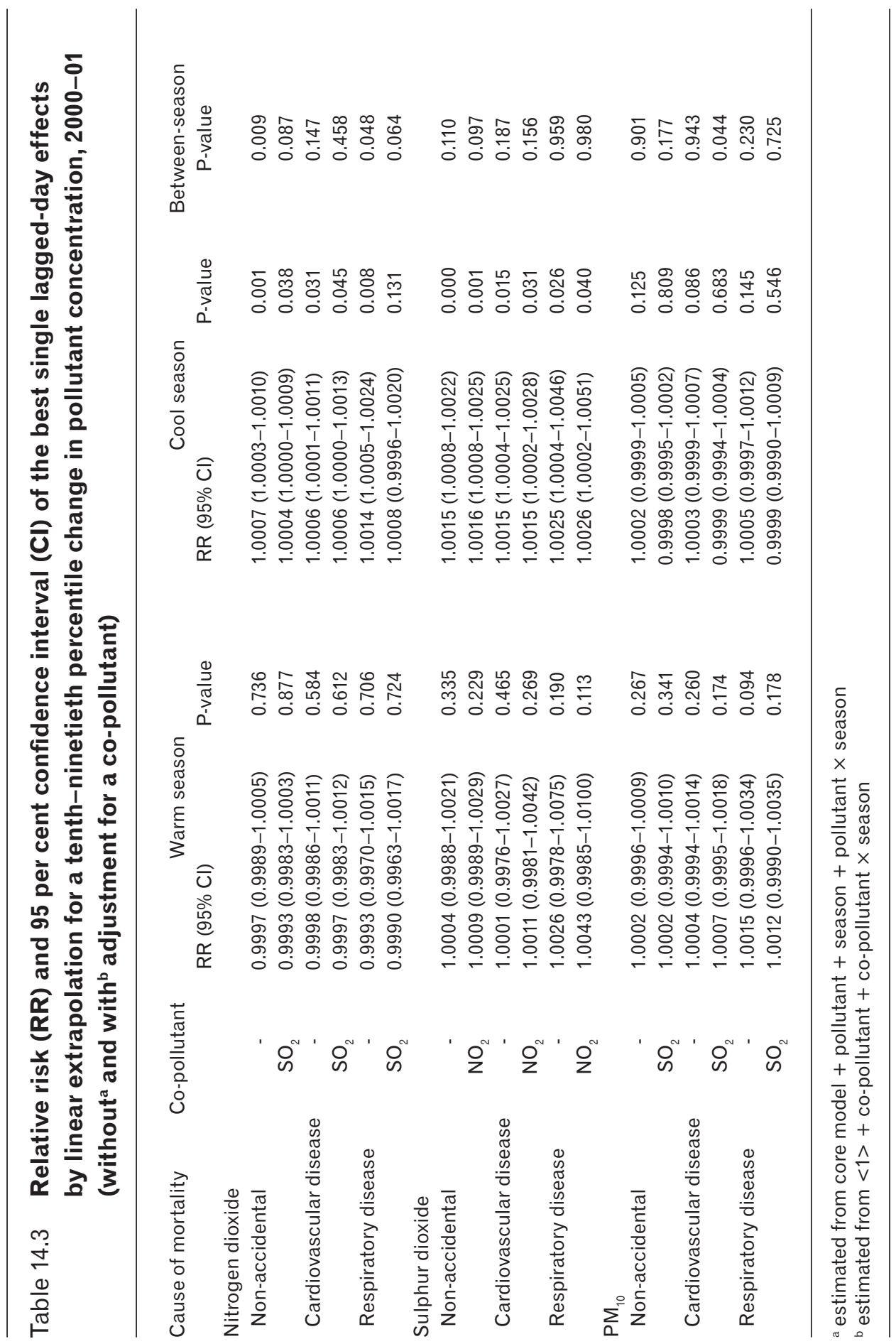


for co-pollutants. As indicated by the statistics shown in the last column of Table 14.3, the between-season differences are statistically significant in estimating the effect of nitrogen dioxide on all non-accidental deaths and deaths caused by respiratory diseases if the impact of the co-pollutant is not adjusted. The between-season difference is also significant when the effect of $\mathrm{PM}_{10}$ on deaths due to cardiovascular diseases is estimated with no adjustment for the co-pollutant.

\section{Discussion}

The results reported in the previous section show that air pollution in Shanghai has a notable impact on population health and mortality. This is particularly the case for sulphur dioxide, which, after adjusting for auto-correlation and for copollutants, still significantly affects the relative risk for all non-accidental deaths and those caused by respiratory diseases. The health impact of air pollution is more observable in cold months, when the level of pollution concentration is markedly higher, than in warm months. As indicated by the results, in the cold season, the positive exposure-response relationships between sulphur dioxide, nitrogen dioxide and specified mortality outcomes are much stronger than in the warm season. These results are supported by evidence presented by other researchers, despite the variation in the reported magnitude of such effects between different studies (Kan et al. 2007; Chen et al. 2007). Epidemiological and medical research have also found that lower temperatures are associated with an increase in hospital admissions for congestive heart failure. 'Both lower temperatures and high air pollutant concentrations were related to increased blood viscosity. Changes in blood rheology may be caused by an inflammatory process in the lung induced by air pollution or by thermoregulatory adjustment to mild surface cooling in cold weather' (Wong et al. 2001:338; see also Keatinge et al. 1984; Peters et al. 1997; Tsai et al. 2003).

Shanghai's municipal government has made some progress in controlling air pollution. Our analysis of air quality in Shanghai between 1998 and 2007 shows that the concentration of $\mathrm{PM}_{10}$ reached its peak in 2003 and started to decline thereafter. The level of nitrogen dioxide also displayed a trend of slow decrease despite a rapid increase in the number of motor vehicles. The level of sulphur dioxide, however, seems to have increased slightly in recent years; this is particularly noteworthy given the adverse health impacts of sulphur dioxide. Further improving its air quality will not only make Shanghai a clean city, it will contribute greatly to improvements in the health of its population. Brajer and Mead (2004) recently estimated the number of deaths that could be averted through controlling air pollution in Chinese cities, including Shanghai. 
While their estimation procedures are questionable, the issues addressed in their paper certainly deserve some attention.

While the conclusions drawn from this study are broadly similar to those reported by the study conducted in Hong Kong by Wong et al. (2001), there are a few differences in the results. For example, while the concentrations of sulphur dioxide and nitrogen dioxide (after controlling for auto-regression and co-pollutants) in both cities have a marked impact on major mortality outcomes, especially in the cold season, the best lag days of such impact are not the same. The impact of sulphur dioxide on major mortality outcomes is readily observable in both cities, but the level of its concentration is more closely related to deaths caused by respiratory diseases in Shanghai, while it shows a stronger association with deaths due to cardiovascular disease in Hong Kong. Furthermore, the health impact of the concentration of nitrogen dioxide is similar in the two cities in the cold season, but it has a stronger association with deaths caused by cardiovascular diseases in Hong Kong if the impact of the season is not taken into consideration in the model. Some of these differences can be explained by variations in the levels of air pollution between Shanghai and Hong Kong; others might be related to the different interaction between various climatic and environmental factors in the two cities-or the selection and specification of statistical models. These possibilities will be examined in our future investigations.

The results reported in this chapter were obtained from our preliminary data analysis of death records and weather and air-quality data gathered in Shanghai in 2000 and 2001. To make the results comparable with those reported by Wong et al. (2001) in their study conducted in Hong Kong, we have applied a method similar to the one they used. This approach has some advantages in facilitating comparative research, but it might have introduced or imposed some restrictions and limitations, especially in the selection and application of the most appropriate statistical methods that could be used effectively to examine the questions under investigation and to analyse the large amount of data collected from Shanghai. We are now exploring the possibility of using more effective statistical methods to analyse the data, and hope this will lead to a more comprehensive examination and contribute to a better understanding of the relationship between weather, air quality, population health and mortality. 


\section{References}

Brajer, V. and Mead, R.W., 2004. 'Valuing air pollution mortality in China's cities', Urban Studies, 41(8):1567-85.

Chen, B., Kan, H. and Zhang, Y., 2007. 'Energy, air pollution and health: a case study in Shanghai', Japanese Journal of Hygiene, 62(2):125-9.

Erbas, B. and Hyndman, R.J., 2005. 'Sensitivity of the estimated air pollutionrespiratory admission relationship to statistical model choice', International Journal of Environmental Health Research, 15(6):437-48.

Kan, H., Chen, B. and Jia, J., 2003. 'A case-crossover study of ambient air pollution and daily mortality in Shanghai', Chinese Journal of Epidemiology (in Chinese), 24(10):863-7.

Kan, H., London, S.J., Chen, G., Zhang, Y., Song, G., Zhao, N., Jiang, L. and Chen, B., 2007. 'Differentiating the effects of fine and coarse particles on daily mortality in Shanghai, China', Environment International, 33(3):376-84.

Keatinge, W.R., Coleshaw, S.R.K., Cotter, F., Mattock, M., Murphy, M. and Chelliah, R., 1984. 'Increases in platelet and red cell counts, blood viscosity, and arterial pressure during mild surface cooling: factors in mortality from coronary and cerebral thrombosis in winter', British Medical Journal (Clinical Research Edition), 289:1405-8.

Kenney, P.L. and Ozkaynak, H., 1991. 'Associations of daily mortality and air pollution in Los Angeles County', Environmental Research, 54(2):99-120.

Lee, J., Shin, D. and Chung, Y., 1999. 'Air pollution and daily mortality in Seoul and Ulsan, Korea', Environmental Health Perspectives, 107(2):149-54.

Ostro, B., Sanchez, J.M., Aranda, C. and Eskeland, G.S., 1996. 'Air pollution and mortality: results from a study of Santiago, Chile', Journal of Exposure Analysis and Environmental Epidemiology, 6(1):97-114.

Peters, A., Doring, A., Wichmann, H.E. and Keonig, W., 1997. 'Increased plasma viscosity during an air pollution episode: a link to mortality?', Lancet, 349:1582-7.

Touloumi, G., Katsouyanni, K., Zmirou, D., Schwartz, J., Spix, C., Ponce de Leon, A., Tobias, A., Quennel, P., Rabcsenko, D. and Bacharova, L., 1997. 'Short-term effects of ambient oxidant exposure on mortality: a combined analysis within the APHEA project', American Journal of Epidemiology, 146(2):177-85.

Tsai, S., Goggins, W., Chiu, H. and Yang, C., 2003. 'Evidence for an association between air pollution and daily stroke admissions in Kaohsiung, Taiwan', Stroke, 34:2612-16.

Wong, C., Ma, S., Hedley, A.J. and Lam, T., 2001. 'Effect of air pollution on daily mortality in Hong Kong', Environmental Health Perspectives, 109(4):335-40. 
World Health Organization (WHO), 2005. World Health Organization air quality guidelines global update 2005, Report on a Working Group Meeting, Bonn, Germany, 18-20 October.

Xu, X., Gao, J., Dockery, D.W. and Chen, Y., 1994. 'Air pollution and daily mortality in residential areas of Beijing, China', Archives of Environmental Health, 49(4):216-22.

Xu, Z., Yu, D., Jing, L. and Xu, X., 2000. 'Air pollution and daily mortality in Shenyang, China', Archives of Environmental Health, 55(2):115-20.

\section{Acknowledgments}

Researchers involved in preparing this chapter include Zhongwei Zhao, Xizhe Peng, Yuan Cheng, Xuehui Han, Guixiang Song, Feng Zhou, Yuhua Shi and Richard Smith. Correspondence should be directed to Professor Zhongwei Zhao, Australian Demographic and Social Research Institute, The Australian National University, ACT 0200, Australia. The Shanghai Mortality Research Team would like to thank the Wellcome Trust for its support for this project (Project Reference No.070318). We would also like to thank Pfizer Inc. and the Hong Kong Research Grants Council for their support for the research. 